\title{
Semantics and Proof Theory of the Epsilon Calculus
}

\author{
Richard Zach ${ }^{\star}$ \\ Department of Philosophy, University of Calgary, Canada \\ rzach@ucalgary.ca
}

\begin{abstract}
The epsilon operator is a term-forming operator which replaces quantifiers in ordinary predicate logic. The application of this undervalued formalism has been hampered by the absence of well-behaved proof systems on the one hand, and accessible presentations of its theory on the other. One significant early result for the original axiomatic proof system for the $\varepsilon$-calculus is the first epsilon theorem, for which a proof is sketched. The system itself is discussed, also relative to possible semantic interpretations. The problems facing the development of proof-theoretically well-behaved systems are outlined.
\end{abstract}

\section{Introduction}

A formalism for logical choice operators has long been available in the form of Hilbert's epsilon calculus. The epsilon calculus is one of the first formal systems of first-order predicate logic. It was introduced in 1921 by David Hilbert [10], who proposed to use it for the formalization and proof theoretical investigation of mathematical systems. In the epsilon calculus, a term-forming operator $\varepsilon$ is used, the intuitive meaning of which is an indefinite choice function: $\varepsilon_{x} A(x)$ is some $x$ which satisfies $A(x)$ if $A(x)$ is satisfied at all, and arbitrary otherwise. Quantifiers can then be defined, e.g., $(\exists x) A(x)$ as $A\left(\epsilon_{x} A(x)\right)$.

The epsilon calculus and proof theoretic methods developed for it, such as the so-called epsilon substitution method, have mainly been applied to the proof theoretic analysis of mathematical systems of arithmetic and analysis (especially in work by Ackermann, Mints, Arai). (See [4] for a survey of the epsilon calculus and its history.) Despite its long history and manifold uses, the epsilon calculus as a logical formalism in general is not thoroughly understood, yet its potential for applications in logic and other areas, especially linguistics and computer science, has by far not been fully explored.

There are various options for definitions of semantics of the epsilon operator. The choice of $\varepsilon_{x} A(x)$ may be extensional (i.e., depend only on the set of $x$ which satisfy $A(x)$; this definition validates the so-called axiom of $\varepsilon$-extensionality), it may be intensional (i.e., depend also on $A(x)$ itself; $\varepsilon$-extensionality fails), and it may be completely indeterministic (i.e., different occurrences of the same $\varepsilon$-term $\varepsilon_{x} A(x)$ may select different witnesses for $\left.A(x)\right)$. The first and third versions

\footnotetext{
* Research supported by the Natural Sciences and Engineering Research Council.
} 
have been investigated by Blass and Gurevich [6]. These different semantics result in different expressive power (in particular, over finite models), and are characterized by different formalizations. Below we present the first two versions of the semantics of the $\varepsilon$-calculus and sketch completeness results.

The very beginnings of proof theory in the work of Hilbert and his students consisted in the proof theoretic study of axiom systems for the $\varepsilon$-calculus. One of the most significant results in this connection are the epsilon theorems. It plays a role similar to Gentzen's midsequent theorem in the proof theory of the sequent calculus: it yields a version of Herbrand's Theorem. In fact, it was used to give the first correct proof of Herbrand's theorem (Hilbert and Bernays [11]). In a simple formulation, the theorem states that if an existential formula $(\exists x) A(x)$ (not containing $\varepsilon$ ) is derivable in the epsilon calculus, then there are terms $t_{1}, \ldots, t_{n}$ so that a (Herbrand-) disjunction $A\left(t_{1}\right) \vee \ldots \vee A\left(t_{n}\right)$ is derivable in propositional logic. The proof gives a constructive procedure that, given a derivation of $(\exists x) A(x)$, produces the corresponding Herbrand disjunction. An analysis of this proof (see [18]) gives a hyper-exponential bound on the length of the Herbrand disjunction in the number of critical formulas occurring in the proof. The bound is essentially optimal, since it is known from work by Orevkov and Statman that the length of Herbrand disjunctions is hyper-exponential in the length of proofs of the original existential formula (this is the basis for familiar speed-up theorems of systems with cut over cut-free systems). In section 4 we prove the first epsilon theorem with identity, along the lines of Bernays's proof.

A general proof theory of the epsilon calculus requires formal systems that are more amenable to proof-theoretic investigations than the Hilbert-type axiomatic systems studied in the Hilbert school. Although some sequent systems for the epsilon calculus exist, it is not clear that they are the best possible formulations, nor have their proof-theoretic properties been investigated in depth. Maehara's [13] and Leisenring's [12] systems were not cut-free complete. Yasuhara [21] studied a cut-free complete system, but only gave a semantic cut-elimination proof. Section 5 surveys these and other systems, and highlights some of the difficulties in developing a systematic proof theory on the basis of them. Proof-theoretically suitable formalisms for the $\varepsilon$-calculus are still a desideratum for applications of the epsilon calculus.

The classical $\varepsilon$-calculus is usually investigated as a proof-theoretic formalism, and no systematic study of the model theory of epsilon calculi other than Asser's classic [3] exists. However, Abiteboul and Vianu [2], Blass and Gurevich [6], and Otto [19] have studied the model theory of choice operators in the context of finite model theory and database query languages. And applications of choice operators to model definite and indefinite noun phrases in computational linguistics Meyer Viol [15] and von Heusinger [8,9] have led to the definition of indexed epsilon calculus by Mints and Sarenac [16].

With a view to applications, it is especially important to develop the semantics and proof theory of epsilon operators in non-classical logics. Of particular importance in this context is the development of epsilon calculi for intuitionistic logic, not least because this is the context in which the epsilon calculus can and 
has been applied in programming language semantics. Some work has been done on intuitionistic $\varepsilon$-calculi (e.g., Bell [5], DeVidi [7], Meyer Viol [15], Mints [17]), but there are still many important open questions. The straightforward extensions of intuitionistic logic by epsilon operators are not conservative and result in intermediate logics related to Gödel logic. Meyer Viol [15] has proposed a conservative extensions of intuitionistic logic by epsilon operators which warrants further study.

\section{Syntax and Axiomatic Proof Systems}

Definition 1. The language of of the elementary calculus $L_{\overline{\mathrm{E}} \mathrm{C}}$ contains the usual logical symbols (variables, function and predicate symbols, $=$ ). A subscript $\varepsilon$ will indicate the presence of the symbol $\varepsilon$, and $\forall$ the presence of the quantifiers $\forall$ and $\exists$. The terms Trm and formulas Frm of $L_{\varepsilon \forall}$ are defined as usual, but simultaneously, to include:

If $A$ is a formula in which $x$ has a free occurrence but no bound occur-

rence, then $\varepsilon_{x} A$ is a term, and all occurrences of $x$ in it are bound.

If $E$ is an expression (term or formula), then $\mathrm{FV}(E)$ is the set of variables which have free occurrences in $E$.

When $E, E^{\prime}$ are expressions (terms or formulas), we write $E \equiv E^{\prime}$ iff $E$ and $E^{\prime}$ are syntactically identical up to a renaming of bound variables. We say that a term $t$ is free for $x$ in $E$ iff $x$ does not occur free in the scope of an $\varepsilon$-operator $\varepsilon_{y}$ or quantifier $\forall y, \exists y$ for any $y \in \mathrm{FV}(t)$.

If $E$ is an expression and $t$ is a term, we write $E[x / t]$ for the result of substituting every free occurrence of $x$ in $E$ by $t$, provided $t$ is free for $x$ in $E$, and renaming bound variables in $t$ if necessary. We write $E(x)$ to indicate that $x \in \mathrm{FV}(E)$, and $E(t)$ for $E[x / t]$. We write $E\{t / u\}$ for the result of replacing every occurrence of $t$ in $E$ by $u .^{1}$

Definition 2 ( $\varepsilon$-Translation). If $E$ is an expression, define $E^{\varepsilon}$ by:

1. $E^{\varepsilon}=E$ if $E$ is a variable, a constant symbol, or $\perp$.

2. If $E=f_{i}^{n}\left(t_{1}, \ldots, t_{n}\right), E^{\varepsilon}=f_{i}^{n}\left(t_{1}^{\varepsilon}, \ldots, t_{n}^{\varepsilon}\right)$.

3. If $E=P_{i}^{n}\left(t_{1}, \ldots, t_{n}\right), E^{\varepsilon}=P_{i}^{n}\left(t_{1}^{\varepsilon}, \ldots, t_{n}^{\varepsilon}\right)$.

4. If $E=\neg A$, then $E^{\varepsilon}=\neg A^{\varepsilon}$.

5. If $E=(A \wedge B),(A \vee B),(A \rightarrow B)$, or $(A \leftrightarrow B)$, then $E^{\varepsilon}=\left(A^{\varepsilon} \wedge B^{\varepsilon}\right)$, $\left(A^{\varepsilon} \vee B^{\varepsilon}\right),\left(A^{\varepsilon} \rightarrow B^{\varepsilon}\right)$, or $\left(A^{\varepsilon} \leftrightarrow B^{\varepsilon}\right)$, respectively.

\footnotetext{
${ }^{1}$ Skipping details, (a) we want to replace not just every occurrence of $t$ by $u$, but every occurrence of a term $t^{\prime} \equiv t$. (b) $t$ may have an occurrence in $E$ where a variable in $t$ is bound by a quantifier or $\varepsilon$ outside $t$, and such occurrences shouldn't be replaced (they are not subterm occurrences). (c) When replacing $t$ by $u$, bound variables in $u$ might have to be renamed to avoid conflicts with the bound variables in $E^{\prime}$ and bound variables in $E^{\prime}$ might have to be renamed to avoid free variables in $u$ being bound.
} 
6. If $E=\exists x A(x)$ or $\forall x A(x)$, then $E^{\varepsilon}=A^{\varepsilon}\left(\varepsilon_{x} A(x)^{\varepsilon}\right)$ or $A^{\varepsilon}\left(\varepsilon_{x} \neg A(x)^{\varepsilon}\right)$.

7. If $E=\varepsilon_{x} A(x)$, then $E^{\varepsilon}=\varepsilon_{x} A(x)^{\varepsilon}$.

Definition 3. An $\varepsilon$-term $p \equiv \varepsilon_{x} B\left(x ; x_{1}, \ldots, x_{n}\right)$ is a type of an $\varepsilon$-term $\varepsilon_{x} A(x)$ iff

1. $p \equiv \varepsilon_{x} A(x)\left[x_{1} / t_{1}\right] \ldots\left[x_{n} / t_{n}\right]$ for some terms $t_{1}, \ldots, t_{n}$.

2. $\mathrm{FV}(p)=\left\{x_{1}, \ldots, x_{n}\right\}$.

3. $x_{1}, \ldots, x_{n}$ are all immediate subterms of $p$.

4. Each $x_{i}$ has exactly one occurrence in $p$.

5. The occurrence of $x_{i}$ is left of the occurrence of $x_{j}$ in $p$ if $i<j$.

We denote the set of types as Typ.

Proposition 4. The type of an epsilon term $\varepsilon_{x} A(x)$ is unique up to renaming of bound, and disjoint renaming of free variables.

Definition 5. An $\varepsilon$-term $e$ is nested in an $\varepsilon$-term $e^{\prime}$ if $e$ is a proper subterm of $e$.

Definition 6. The degree $\operatorname{deg}(e)$ of an $\varepsilon$-term $e$ is defined as follows: $(1) \operatorname{deg}(e)=$ 1 iff $e$ contains no nested $\varepsilon$-terms. (2) $\operatorname{deg}(e)=\max \left\{\operatorname{deg}\left(e_{1}\right), \ldots, \operatorname{deg}\left(e_{n}\right)\right\}+1$ if $e_{1}, \ldots, e_{n}$ are all the $\varepsilon$-terms nested in $e$. For convenience, let $\operatorname{deg}(t)=0$ if $t$ is not an $\varepsilon$-term.

Definition 7. An $\varepsilon$-term $e$ is subordinate to an $\varepsilon$-term $e^{\prime}=\varepsilon_{x} A(x)$ if some $e^{\prime \prime} \equiv e$ occurs in $e^{\prime}$ and $x \in \mathrm{FV}\left(e^{\prime \prime}\right)$.

Note that if $e$ is subordinate to $e^{\prime}$ it is not a subterm of $e^{\prime}$, because $x$ is free in $e$ and so the occurrence of $e$ (really, of the variant $e^{\prime \prime}$ ) in $e^{\prime}$ is in the scope of $\varepsilon_{x} \cdot{ }^{2}$

Definition 8. The $\operatorname{rank} \operatorname{rk}(e)$ of an $\varepsilon$-term $e$ is defined as follows: $(1) \operatorname{rk}(e)=1$ iff $e$ contains no subordinate $\varepsilon$-terms. (2) $\operatorname{rk}(e)=\max \left\{\operatorname{rk}\left(e_{1}\right), \ldots, \operatorname{rk}\left(e_{n}\right)\right\}+1$ if $e_{1}, \ldots, e_{n}$ are all the $\varepsilon$-terms subordinate to $e$.

Proposition 9. If $p$ is the type of e, then $\operatorname{rk}(p)=\operatorname{rk}(e)$.

\subsection{Axioms and Proofs}

Definition 10. The axioms of the elementary calculus EC are

$A$ for any tautology $A$

and its only rule of inference is

$$
\frac{A \quad A \rightarrow B}{A} \mathrm{MP}
$$

\footnotetext{
${ }^{2}$ One might think that replacing $e$ in $\varepsilon_{x} A(x)$ by a new variable $y$ would result in an $\varepsilon$-term $\varepsilon_{x} A^{\prime}(y)$ so that $e^{\prime} \equiv \varepsilon_{x} A^{\prime}(y)[y / e]$. But (a) $\varepsilon_{x} A^{\prime}(y)$ is not in general a term, since it is not guaranteed that $x$ is free in $A^{\prime}(y)$ and (b) $e$ is not free for $y$ in $\varepsilon_{x} A^{\prime}(y)$.
} 
For $\mathrm{EC}^{=}$, we add

$$
\begin{aligned}
& t=t \\
& t=u \rightarrow(A[x / t] \leftrightarrow A[x / u]) .
\end{aligned}
$$

The axioms and rules of the (intensional) $\varepsilon$-calculus $\mathrm{EC}_{\varepsilon}\left(\mathrm{EC}_{\varepsilon}^{=}\right)$are those of $\mathrm{EC}$ $\left(\mathrm{EC}^{=}\right)$plus the critical formulas

$$
A(t) \rightarrow A\left(\varepsilon_{x} A(x)\right) .
$$

The axioms and rules of the extensional $\varepsilon$-calculus $\mathrm{EC}_{\varepsilon}^{\text {ext }}$ are those of $\mathrm{EC}_{\varepsilon}^{=}$plus

$$
(\forall x(A(x) \leftrightarrow B(x)))^{\varepsilon} \rightarrow \varepsilon_{x} A(x)=\varepsilon_{x} B(x), \quad(\mathrm{ext})
$$

that is,

$$
A\left(\varepsilon_{x} \neg(A(x) \leftrightarrow B(x))\right) \leftrightarrow B\left(\varepsilon_{x} \neg(A(x) \leftrightarrow B(x))\right) \rightarrow \varepsilon_{x} A(x)=\varepsilon_{x} B(x)
$$

The axioms and rules of $\mathrm{EC}_{\forall}, \mathrm{EC}_{\varepsilon \forall}, \mathrm{EC}_{\varepsilon \forall}^{\text {ext }}$ are those of $\mathrm{EC}, \mathrm{EC}_{\varepsilon}, \mathrm{EC}_{\varepsilon}^{\text {ext }}$, respectively, together with the axioms

$$
\begin{aligned}
A(t) & \rightarrow \exists x A(x) \\
\forall x A(x) & \rightarrow A(t)
\end{aligned}
$$

and the rules

$$
\frac{A(x) \rightarrow B}{\exists x A(x) \rightarrow B} R \exists \quad \frac{B \rightarrow A(x)}{B \rightarrow \forall x A(x)} R \forall
$$

Applications of these rules must satisfy the eigenvariable condition, viz., the variable $x$ must not appear in the conclusion or anywhere below it in the proof.

Definition 11. If $\Gamma$ is a set of formulas, a proof of $A$ from $\Gamma$ in $\mathrm{EC}_{\varepsilon \forall}^{\text {ext }}$ is a sequence $\pi$ of formulas $A_{1}, \ldots, A_{n}=A$ where for each $i \leq n, A_{i} \in \Gamma, A_{i}$ is an instance of an axiom, or follows from formulas $A_{j}(j<i)$ by a rule of inference.

If $\pi$ only uses the axioms and rules of $\mathrm{EC}, \mathrm{EC}_{\varepsilon}, \mathrm{EC}_{\varepsilon}^{\text {ext }}$, etc., then it is a proof of $A$ from $\Gamma$ in $\mathrm{EC}, \mathrm{EC}_{\varepsilon}, \mathrm{EC}_{\varepsilon}^{\text {ext }}$, etc., and we write $\Gamma \vdash^{\pi} A, \Gamma \vdash_{\varepsilon}^{\pi} A, \Gamma \vdash_{\varepsilon \text { ext }}^{\pi} A$, etc.

We say that $A$ is provable from $\Gamma$ in EC, etc. $(\Gamma \vdash A$, etc.), if there is a proof of $A$ from $\Gamma$ in EC, etc.

Note that our definition of proof, because of its use of $\equiv$, includes a tacit rule for renaming bound variables. Note also that substitution into members of $\Gamma$ is not permitted. However, we can simulate a provability relation in which substitution into members of $\Gamma$ is allowed by considering $\Gamma^{\text {inst }}$, the set of all substitution instances of members of $\Gamma$. If $\Gamma$ is a set of sentences, then $\Gamma^{\text {inst }}=\Gamma$.

Proposition 12. If $\pi=A_{1}, \ldots, A_{n} \equiv A$ is a proof of $A$ from $\Gamma$ and $x \notin \mathrm{FV}(\Gamma)$ is not an eigenvariable in $\pi$, then $\pi[x / t]=A_{1}[x / t], \ldots, A_{n}[x / t]$ is a proof of $A[x / t]$ from $\Gamma^{\text {inst }}$. 
Lemma 13. If $\pi$ is a proof of $B$ from $\Gamma \cup\{A\}$, then there is a proof $\pi[A]$ of $A \rightarrow B$ from $\Gamma$, provided $A$ contains no eigenvariables of $\pi$ free.

Proof. By induction on the length of $\pi$, as in the classical case.

Theorem 14 (Deduction Theorem). If $\Sigma \cup\{A\}$ is a set of sentences, $\Sigma \vdash$ $A \rightarrow B$ iff $\Sigma \cup\{A\} \vdash B$.

Corollary 15. If $\Sigma \cup\{A\}$ is a set of sentences, $\Sigma \vdash A$ iff $\Sigma \cup\{\neg A\} \vdash \perp$.

Lemma 16 ( $\varepsilon$-Embedding Lemma). If $\Gamma \vdash_{\varepsilon \forall}^{\pi} A$, then there is a proof $\pi^{\varepsilon}$ so that $\Gamma^{\varepsilon i n s t} \vdash_{\varepsilon}^{\pi^{\varepsilon}} A^{\varepsilon}$

Proof. By induction, see [18].

\section{Semantics and Completeness}

\subsection{Semantics for $\mathrm{EC}_{\varepsilon}^{\mathrm{ext}}$}

Definition 17. A structure $\mathfrak{M}=\left\langle|\mathfrak{M}|,(\cdot)^{\mathfrak{M}}\right\rangle$ consists of a nonempty domain $|\mathfrak{M}| \neq$ $\emptyset$ and a mapping $(\cdot)^{\mathfrak{M}}$ on function and predicate symbols where $\left(f_{i}^{0}\right)^{\mathfrak{M}} \in|\mathfrak{M}|$, $\left(f_{i}^{n}\right)^{M} \in|\mathfrak{M}|^{|\mathfrak{M}|^{n}}$, and $\left(P_{i}^{n}\right)^{\mathfrak{M}} \subseteq|\mathfrak{M}|^{n}$.

Definition 18. An extensional choice function $\Phi$ on $\mathfrak{M}$ is a function $\Phi: \wp(|\mathfrak{M}|) \rightarrow$ $|\mathfrak{M}|$ where $\Phi(X) \in X$ whenever $X \neq \emptyset$.

Note that $\Phi$ is total on $\wp(|\mathfrak{M}|)$, and so $\Phi(\emptyset) \in|\mathfrak{M}|$.

Definition 19. An assignment $s$ on $\mathfrak{M}$ is a function $s$ : Var $\rightarrow|\mathfrak{M}|$.

If $x \in$ Var and $m \in|\mathfrak{M}|, s[x / m]$ is the assignment defined by

$$
s[x / m](y)= \begin{cases}m & \text { if } y=x \\ s(y) & \text { otherwise }\end{cases}
$$

Definition 20. The value $\operatorname{val}_{\mathfrak{M}, \Phi, s}(t)$ of a term and the satisfaction relation $\mathfrak{M}, \Phi, s \models A$ are defined as follows:

1. $\operatorname{val}_{\mathfrak{M}, \Phi, s}(x)=s(x)$

2. $\mathfrak{M}, \Phi, s \models \top$ and $\mathfrak{M}, \Phi, s \not \models \perp$

3. $\operatorname{val}_{\mathfrak{M}, \Phi, s}\left(f_{i}^{n}\left(t_{1}, \ldots, t_{n}\right)\right)=\left(f_{i}^{n}\right)^{\mathfrak{M}}\left(\operatorname{val}_{\mathfrak{M}, \Phi, s}\left(t_{1}\right), \ldots, \operatorname{val}_{\mathfrak{M}, \Phi, s}\left(t_{n}\right)\right)$

4. $\mathfrak{M}, \Phi, s \models t_{1}=t_{n}$ iff $\operatorname{val}_{\mathfrak{M}, \Phi, s}\left(t_{1}\right)=\operatorname{val}_{\mathfrak{M}, \Phi, s}\left(t_{2}\right)$

5. $\mathfrak{M}, \Phi, s \models P_{i}^{n}\left(t_{1}, \ldots, t_{n}\right)$ iff $\left\langle\operatorname{val}_{\mathfrak{M}, \Phi, s}\left(t_{1}\right), \ldots, \operatorname{val}_{\mathfrak{M}, \Phi, s}\left(t_{n}\right)\right\rangle \in\left(P_{i}^{n}\right)^{\mathfrak{M}}$

6. $\operatorname{val}_{\mathfrak{M}, \Phi, s}\left(\varepsilon_{x} A(x)\right)=\Phi\left(\operatorname{val}_{\mathfrak{M}, \Phi, s}(A(x))\right)$ where

$$
\operatorname{val}_{\mathfrak{M}, \Phi, s}(A(x))=\{m \in|\mathfrak{M}|: \mathfrak{M}, \Phi, s[x / m] \models A(x)\}
$$

7. $\mathfrak{M}, \Phi, s \models \exists x A(x)$ iff for some $m \in|\mathfrak{M}|, \mathfrak{M}, \Phi, s[x / m] \models A(x)$

8. $\mathfrak{M}, \Phi, s \models \forall x A(x)$ iff for all $m \in|\mathfrak{M}|, \mathfrak{M}, \Phi, s[x / m] \models A(x)$ 
Proposition 21. If $s(x)=s^{\prime}(x)$ for all $x \notin \mathrm{FV}(t) \cup \mathrm{FV}(A)$, then $\operatorname{val}_{\mathfrak{M}, \Phi, s}(t)=$ $\operatorname{val}_{\mathfrak{M}, \Phi, s^{\prime}}(t)$ and $\mathfrak{M}, \Phi, s \models A$ iff $\mathfrak{M}, \Phi, s^{\prime} \models A$.

Proposition 22 (Substitution Lemma). If $m=\operatorname{val}_{\mathfrak{M}, \Phi, s}(u)$, then $\operatorname{val}_{\mathfrak{M}, \Phi, s}(t(u))=$ $\operatorname{val}_{\mathfrak{M}, \Phi, s[x / m]}(t(x))$ and $\mathfrak{M}, \Phi, s \models A(u)$ iff $\mathfrak{M}, \Phi, s[x / m] \models A(x)$

Definition 23. 1. $A$ is locally true in $\mathfrak{M}$ w.r.t. $\Phi$ and $s$ iff $\mathfrak{M}, \Phi, s \models A$.

2. $A$ is true in $\mathfrak{M}$ with respect to $\Phi, \mathfrak{M}, \Phi \models A$, iff for all $s$ on $\mathfrak{M}: \mathfrak{M}, \Phi, s \models A$.

3. $A$ is generically true in $\mathfrak{M}$ with respect to $s, \mathfrak{M}, s \models^{g} A$, iff for all choice functions $\Phi$ on $\mathfrak{M}: \mathfrak{M}, \Phi, s \models A$.

4. $A$ is generically valid in $\mathfrak{M}, \mathfrak{M} \models A$, if for all choice functions $\Phi$ and assignments $s$ on $\mathfrak{M}: \mathfrak{M}, \Phi, s \models A$.

Definition 24. Let $\Gamma \cup\{A\}$ be a set of formulas.

1. $A$ is a local consequence of $\Gamma, \Gamma \models^{l} A$, iff for all $\mathfrak{M}, \Phi$, and $s$ : if $\mathfrak{M}, \Phi, s \models \Gamma$ then $\mathfrak{M}, \Phi, s \models A$.

2. $A$ is a truth consequence of $\Gamma, \Gamma \models A$, iff for all $\mathfrak{M}$, $\Phi$ : if $\mathfrak{M}, \Phi \models \Gamma$ then $\mathfrak{M}, \Phi \models A$.

3. $A$ is a generic consequence of $\Gamma, \Gamma \models^{g} A$, iff for all $\mathfrak{M}$ and $s$ : if $\mathfrak{M}, s \models^{g} \Gamma$ then $\mathfrak{M} \models A$.

4. $A$ is a generic validity consequence of $\Gamma, \Gamma \models^{v} A$, iff for all $\mathfrak{M}$ : if $\mathfrak{M} \models^{v} \Gamma$ then $\mathfrak{M} \models A$.

Proposition 25. If $\Sigma \cup\{A\}$ is a set of sentences, $\Sigma \models^{l} A$ iff $\Sigma \models A$

Proposition 26. If $\Sigma \cup\{A, B\}$ is a set of sentences, $\Sigma \cup\{A\} \models B$ iff $\Sigma \models$ $A \rightarrow B$.

Corollary 27. If $\Sigma \cup\{A\}$ is a set of sentences, $\Sigma \models A$ iff for no $\mathfrak{M}, \Phi, \mathfrak{M} \models$ $\Sigma \cup\{\neg A\}$

\subsection{Soundness and Completeness}

Theorem 28. If $\Gamma \vdash_{\varepsilon} A$, then $\Gamma \models^{l} A$.

Proof. Suppose $\Gamma, \Phi, s \models \Gamma$. We show by induction on the length $n$ of a proof $\pi$ that $\mathfrak{M}, \Phi, s^{\prime} \models A$ for all $s^{\prime}$ which agree with $s$ on $\operatorname{FV}(\Gamma)$. We may assume that no eigenvariable $x$ of $\pi$ is in $\mathrm{FV}(\Gamma)$ (if it is, let $y \notin \mathrm{FV}(\pi)$ and not occurring in $\pi$; consider $\pi[x / y]$ instead of $\pi$ ).

If $n=0$ there's nothing to prove. Otherwise, we distinguish cases according to the last line $A_{n}$ in $\pi$. The only interesting case is when $A_{n}$ is a critical formula, i.e., $A_{n} \equiv A(t) \rightarrow A\left(\varepsilon_{x} A(x)\right)$. Then either $\mathfrak{M}, \Phi, s \models A(t)$ or not (in which case there's nothing to prove). If yes, $\mathfrak{M}, \Phi, s[x / m] \models A(x)$ for $m=\operatorname{val}_{\mathfrak{M}, \Phi, s}(t)$, and so $Y=\operatorname{val}_{\mathfrak{M}, \Phi, s}(A(x)) \neq \emptyset$. Consequently, $\Phi(Y) \in Y$, and hence $\mathfrak{M}, \Phi, s \models$ $A\left(\varepsilon_{x} A(x)\right)$.

Lemma 29. If $\Gamma$ is a set of sentences and $\Gamma \forall_{\varepsilon} \perp$, then there are $\mathfrak{M}$, $\Phi$ so that $\mathfrak{M}, \Phi \models \Gamma$. 
Theorem 30 (Completeness). If $\Gamma \cup\{A\}$ are sentences and $\Gamma \models A$, then $\Gamma \vdash_{\varepsilon \operatorname{ext}} A$.

Proof. Suppose $\Gamma \not \models A$. Then for some $\mathfrak{M}$, $\Phi$ we have $\mathfrak{M}, \Phi \models \Gamma$ but $\mathfrak{M}, \Phi \not \models A$. Hence $\mathfrak{M}, \Phi \models \Gamma \cup\{\neg A\}$. By the Lemma, $\Gamma \cup\{\neg A\} \vdash_{\varepsilon} \perp$. By Corollary 15, $\Gamma \vdash_{\varepsilon} A$.

The proof of the Lemma comes in several stages. We have to show that if $\Gamma$ is consistent, we can construct $\mathfrak{M}, \Phi$, and $s$ so that $\mathfrak{M}, \Phi, s \models \Gamma$. Since $\operatorname{FV}(\Gamma)=\emptyset$, we then have $\mathfrak{M}, \Phi \models \Gamma$.

Lemma 31. If $\Gamma \nvdash_{\varepsilon} \perp$, there is $\Gamma^{*} \supseteq \Gamma$ with (1) $\Gamma^{*} \nvdash_{\varepsilon} \perp$ and (2) for all formulas $A$, either $A \in \Gamma^{*}$ or $\neg A \in \Gamma^{*}$.

Proof. Let $A_{1}, A_{2}, \ldots$ be an enumeration of $\operatorname{Frm}_{\varepsilon}$. Define $\Gamma_{0}=\Gamma$ and

$$
\Gamma_{n+1}= \begin{cases}\Gamma_{n} \cup\left\{A_{n}\right\} & \text { if } \Gamma_{n} \cup\left\{A_{n}\right\} \forall_{\varepsilon} \perp \\ \Gamma_{n} \cup\left\{\neg A_{n}\right\} & \text { if } \Gamma_{n} \cup\left\{\neg A_{n}\right\} \forall_{\varepsilon} \perp \text { otherwise }\end{cases}
$$

Let $\Gamma^{*}=\bigcup_{n \geq 0} \Gamma_{n}$. Obviously, $\Gamma \subseteq \Gamma^{*}$. For (1), observe that if $\Gamma^{*} \vdash_{\varepsilon}^{\pi} \perp$, then $\pi$ contains only finitely many formulas from $\Gamma^{*}$, so for some $n, \Gamma_{n} \vdash_{\varepsilon}^{\pi} \perp$. But $\Gamma_{n}$ is consistent by definition.

To verify (2), we have to show that for each $n$, either $\Gamma_{n} \cup\left\{A_{n}\right\} \forall_{\varepsilon} \perp$ or $\Gamma_{n} \cup\{\neg A\} \forall \varepsilon \perp$. For $n=0$, this is the assumption of the lemma. So suppose the claim holds for $n-1$. Suppose $\Gamma_{n} \cup\{A\} \vdash_{\varepsilon}^{\pi} \perp$ and $\Gamma_{n} \cup\{\neg A\} \vdash_{\varepsilon}^{\pi^{\prime}} \perp$. Then by the Deduction Theorem, we have $\Gamma_{n} \vdash_{A}^{\pi[A]} \rightarrow \perp$ and $\Gamma_{n} \vdash_{\neg}^{\pi^{\prime}\left[A^{\prime}\right]} A \rightarrow \perp$. Since $(A \rightarrow \perp) \rightarrow((\neg A \rightarrow \perp) \rightarrow \perp)$ is a tautology, we have $\Gamma_{n} \vdash_{\varepsilon} \perp$, contradicting the induction hypothesis.

Lemma 32. If $\Gamma^{*} \vdash_{\varepsilon} B$, then $B \in \Gamma^{*}$.

Proof. If not, then $\neg B \in \Gamma^{*}$ by maximality, so $\Gamma^{*}$ would be inconsistent.

Definition 33. Let $\approx$ be the relation on $\operatorname{Trm}_{\varepsilon}$ defined by

$$
t \approx u \text { iff } t=u \in \Gamma^{*}
$$

It is easily seen that $\approx$ is an equivalence relation. Let $\widetilde{t}=\{u: u \approx t\}$ and $\widetilde{\operatorname{Trm}}=\{\tilde{t}: t \in \operatorname{Trm}\}$.

Definition 34. A set $T \in \widetilde{\operatorname{Trm}}$ is represented by $A(x)$ if $T=\left\{\widetilde{t}: A(t) \in \Gamma^{*}\right\}$.

Let $\Phi_{0}$ be a fixed choice function on $\widetilde{\operatorname{Trm}}$, and define

$$
\Phi(T)= \begin{cases}\widetilde{\varepsilon_{x} A(x)} & \text { if } T \text { is represented by } A(x) \\ \Phi_{0}(T) & \text { otherwise. }\end{cases}
$$

Proposition 35. $\Phi$ is a well-defined choice function on $\widetilde{\text { Trm. }}$. 
Proof. Use (ext) for well-definedness and (crit) for choice function.

Now let $\mathfrak{M}=\left\langle\widetilde{\operatorname{Trm}},(\cdot)^{\mathfrak{M}}\right\rangle$ with $c^{\mathfrak{M}}=\widetilde{c},\left(P_{i}^{n}\right)^{\mathfrak{M}}=\left\{\left\langle\widetilde{t}_{1}, \ldots, \widetilde{t}_{1}\right\rangle: P_{i}^{n}\left(t_{1}, \ldots, t_{n}\right)\right\}$, and let $s(x)=\widetilde{s}$.

Proposition 36. $\mathfrak{M}, \Phi, s \models \Gamma^{*}$.

Proof. We show that $\operatorname{val}_{\mathfrak{M}, \Phi, s}(t)=\widetilde{t}$ and $\mathfrak{M}, \Phi, s \models A$ iff $A \in \Gamma^{*}$ by simultaneous induction on the complexity of $t$ and $A$.

If $t=c$ is a constant, the claim holds by definition of $(\cdot)^{\mathfrak{M}}$. If $A=\perp$ or $=\top$, the claim holds by Lemma 32 .

If $A \equiv P^{n}\left(t_{1}, \ldots, t_{n}\right)$, then by induction hypothesis, val $\widetilde{M}_{\mathfrak{M}, \Phi, s}(t)_{i}={\widetilde{t_{i}}}_{i}$. By definition of $(\cdot)^{\mathfrak{M}},\left\langle\widetilde{t_{1}}, \ldots, \widetilde{t_{n}}\right\rangle \in\left(P_{i}^{n}\right)\left(t_{1}, \ldots, t_{n}\right)$ iff $P_{i}^{n}\left(t_{1}, \ldots, t_{n}\right) \in \Gamma^{*}$.

If $A \equiv \neg B,(B \wedge C),(B \vee C),(B \rightarrow C),(B \leftrightarrow C)$, the claim follows immediately from the induction hypothesis and the definition of $\models$ and the closure properties of $\Gamma^{*}$. For instance, $\mathfrak{M}, \Phi, s \models(B \wedge C)$ iff $\mathfrak{M}, \Phi, s \models B$ and $\mathfrak{M}, \Phi, s \models C$. By induction hypothesis, this is the case iff $B \in \Gamma^{*}$ and $C \in \Gamma^{*}$. But since $B, C \vdash_{\varepsilon} B \wedge C$ and $B \wedge C \vdash_{\varepsilon} B$ and $\vdash_{\varepsilon} C$, this is the case iff $(B \wedge C) \in \Gamma^{*}$. Remaining cases: Exercise.

If $t \equiv \varepsilon_{x} A(x)$, then $\operatorname{val}_{\mathfrak{M}, \Phi, s}(t)=\Phi\left(\operatorname{val}_{\mathfrak{M}, \Phi, s}(A(x))\right)$. Since $\operatorname{val}_{\mathfrak{M}, \Phi, s}(A(x))$ is represented by $A(x)$ by induction hypothesis, we have $\operatorname{val}_{\mathfrak{M}, \Phi, s}(t)=\widetilde{\varepsilon_{x} A(x)}$ by definition of $\Phi$.

\subsection{Semantics for $\mathbf{E C}_{\varepsilon}$}

In order to give a complete semantics for $\mathrm{EC}_{\varepsilon}$, i.e., for the calculus without the extensionality axiom (ext), it is necessary to change the notion of choice function so that two $\varepsilon$-terms $\varepsilon_{x} A(x)$ and $\varepsilon_{x} B(x)$ may be assigned different representatives even when $\mathfrak{M}, \Phi, s \models \forall x(A(x) \leftrightarrow B(x))$, since then the negation of (ext) is consistent in the resulting calculus. The idea is to add the $\varepsilon$-term itself as an additional argument to the choice function. However, in order for this semantics to be sound for the calculus - specifically, in order for $\left(=_{2}\right)$ to be valid - we have to use not $\varepsilon$-terms but $\varepsilon$-types.

Definition 37. An intensional choice operator is a mapping $\Psi$ : Typ $\times|\mathfrak{M}|^{<\omega} \rightarrow$ $|\mathfrak{M}|^{\wp(|\mathfrak{M}|)}$ such that for every type $p=\varepsilon_{x} A\left(x ; y_{1}, \ldots, y_{n}\right)$ is a type, and $m_{1}$, $\ldots, m_{n} \in|\mathfrak{M}|, \Psi\left(p, m_{1}, \ldots, m_{n}\right)$ is a choice function.

Definition 38. If $\mathfrak{M}$ is a structure, $\Psi$ an intensional choice operator, and $s$ an assignment, $\operatorname{val}_{\mathfrak{M}, \Psi, s}(t)$ and $\mathfrak{M}, \Psi, s \models A$ is defined as before, except (6) in Definition 20 is replaced by:

$\left(6^{\prime}\right) \operatorname{val}_{\mathfrak{M}, \Psi, s}\left(\varepsilon_{x} A(x)\right)=\Psi\left(p, m_{1}, \ldots, m_{n}\right)\left(\operatorname{val}_{\mathfrak{M}, \Phi, s}(A(x))\right)$ where

(a) $p=\varepsilon_{x} A^{\prime}\left(x ; x_{1}, \ldots, x_{n}\right)$ is the type of $\varepsilon_{x} A(x)$,

(b) $t_{1}, \ldots, t_{n}$ are the subterms corresponding to $x_{1}, \ldots, x_{n}$, i.e., $\varepsilon_{x} A(x) \equiv$ $\varepsilon_{x} A^{\prime}\left(x ; t_{1}, \ldots, t_{n}\right)$,

(c) $m_{i}=\operatorname{val}_{\mathfrak{M}, \Psi, s}(t)_{1}$, and

(d) $\operatorname{val}_{\mathfrak{M}, \Phi, s}(A(x))=\{m \in|\mathfrak{M}|: \mathfrak{M}, \Psi, s[x / m] \models A(x)\}$

The soundness and completeness proofs generalize to $\mathrm{EC}_{\varepsilon}, \mathrm{EC}_{\varepsilon}^{=}$, and $\mathrm{EC}_{\varepsilon \forall}$. 


\section{The First Epsilon Theorem}

\subsection{The Case Without Identity}

Definition 39. An $\varepsilon$-term $e$ is critical in $\pi$ if $A(t) \rightarrow A(e)$ is one of the critical formulas in $\pi$. The rank $\operatorname{rk}(\pi)$ of a proof $\pi$ is the maximal rank of its critical $\varepsilon$-terms. The $r$-degree $\operatorname{deg}(\pi, r)$ of $\pi$ is the maximum degree of its critical $\varepsilon$-terms of rank $r$. The $r$-order $o(\pi, r)$ of $\pi$ is the number of different (up to renaming of bound variables) critical $\varepsilon$-terms of rank $r$.

Lemma 40. If $e=\varepsilon_{x} A(x), \varepsilon_{y} B(y)$ are critical in $\pi, \operatorname{rk}(e)=\operatorname{rk}(\pi)$, and $B^{*} \equiv$ $B(u) \rightarrow B\left(\varepsilon_{y} B(y)\right)$ is a critical formula in $\pi$. Then, if $e$ is a subterm of $B^{*}$, it is a subterm of $B(y)$ or a subterm of $u$.

Proof. Suppose not. Since $e$ is a subterm of $B^{*}$, we have $B(y) \equiv B^{\prime}\left(\varepsilon_{x} A^{\prime}(x, y), y\right)$ and either $e \equiv \varepsilon_{x} A^{\prime}(x, u)$ or $e \equiv \varepsilon_{x} A^{\prime}\left(x, \varepsilon_{y} B(y)\right)$. In each case, we see that $\varepsilon_{x} A^{\prime}(x, y)$ and $e$ have the same rank, since the latter is an instance of the former (and so have the same type). On the other hand, in either case, $\varepsilon_{y} B(y)$ would be

$$
\varepsilon_{y} B^{\prime}\left(\varepsilon_{x} A^{\prime}(x, y), y\right)
$$

and so would have a higher rank than $\varepsilon_{x} A^{\prime}(x, y)$ as that $\varepsilon$-term is subordinate to it. This contradicts $\operatorname{rk}(e)=\operatorname{rk}(\pi)$.

Lemma 41. Let $e, B^{*}$ be as in the lemma, and $t$ be any term. Then

1. If $e$ is not a subterm of $B(y), B^{*}\{e / t\} \equiv B\left(u^{\prime}\right) \rightarrow B\left(\varepsilon_{y} B(y)\right)$.

2. If $e$ is a subterm of $B(y)$, i.e., $B(y) \equiv B^{\prime}(e, y), B^{*}\{e / t\} \equiv B^{\prime}\left(t, u^{\prime}\right) \rightarrow$ $B^{\prime}\left(t, \varepsilon_{y} B^{\prime}(t, y)\right)$.

Lemma 42. If $\vdash_{\varepsilon}^{\pi} E$ and $E$ does not contain $\varepsilon$, then there is a proof $\pi^{\prime}$ such that $\vdash_{\varepsilon}^{\pi^{\prime}} E$ and $\operatorname{rk}\left(\pi^{\prime}\right) \leq \operatorname{rk}(p i)=r$ and $o\left(\pi^{\prime}, r\right)<o(\pi, r)$.

Proof. Let $e$ be an $\varepsilon$-term critical in $\pi$ and let $A\left(t_{1}\right) \rightarrow A(e)$, dots, $A\left(t_{n}\right) \rightarrow A(e)$ be all its critical formulas in $\pi$.

Consider $\pi\{e / t\}_{i}$, i.e., $\pi$ with $e$ replaced by $t_{i}$ throughout. Each critical formula belonging to $e$ now is of the form $A\left(t_{j}^{\prime}\right) \rightarrow A\left(t_{i}\right)$, since $e$ obviously cannot be a subterm of $A(x)$ (if it were, $e$ would be a subterm of $\varepsilon_{x} A(x)$, i.e., of itself!). Let $\hat{\pi}_{i}$ be the sequence of tautologies $A\left(t_{i}\right) \rightarrow\left(A\left(t_{j}^{\prime}\right) \rightarrow A\left(t_{i}\right)\right)$ for $i=1, \ldots, n$, followed by $\pi\{e / t\}_{i}$. Each one of the formulas $A\left(t_{j}^{\prime}\right) \rightarrow A\left(t_{i}\right)$ follows from one of these by (MP) from $A\left(t_{i}\right)$. Hence, $A\left(t_{i}\right) \vdash_{\varepsilon}^{\hat{\pi}_{i}} E$. Let $\pi_{i}=\hat{\pi}_{i}\left[A_{i}\right]$ as in Lemma 13 . We have $\vdash_{\varepsilon}^{\pi_{i}} A_{i} \rightarrow E$.

The $\varepsilon$-term $e$ is not critical in $\pi_{i}$ : Its original critical formulas are replaced by $A\left(t_{i}\right) \rightarrow\left(A\left(t_{j}^{\prime}\right) \rightarrow A\left(t_{i}\right)\right)$, which are tautologies. By (1) of the preceding Lemma, no critical $\varepsilon$-term of rank $r$ was changed at all. By (2) of the preceding Lemma, no critical $\varepsilon$-term of rank $<r$ was replaced by a critical $\varepsilon$-term of rank $\geq r$. Hence, $o\left(\pi_{i}, r\right)=o(\pi)-1$.

Let $\pi^{\prime \prime}$ be the sequence of tautologies $\neg \bigvee_{i=1}^{n} A\left(t_{i}\right) \rightarrow\left(A\left(t_{i}\right) \rightarrow A(e)\right)$ followed by $\pi$. Then $\bigvee_{i=1}^{n} A\left(t_{i}\right) \vdash_{E}^{\pi^{\prime \prime}}, e$ is not critical in $\pi^{\prime \prime}$, and otherwise $\pi$ and $\pi^{\prime \prime}$ 
have the same critical formulas. The same goes for $\pi^{\prime \prime}\left[\neg \bigvee A\left(t_{i}\right)\right]$, a proof of $\neg \bigvee A\left(t_{i}\right) \rightarrow E$.

We now obtain $\pi^{\prime}$ as the $\pi_{i}, i=1, \ldots, n$, followed by $\pi\left[\neg \bigvee_{i=1}^{n} A\left(t_{i}\right)\right]$, followed by the tautology

$$
\left.\left.\left(\neg \bigvee A\left(t_{i}\right) \rightarrow E\right) \rightarrow\left(A\left(t_{1}\right) \rightarrow E\right) \rightarrow \cdots \rightarrow\left(A\left(t_{n}\right) \rightarrow E\right) \rightarrow E\right) \ldots\right)
$$

from which $E$ follows by $n+1$ applications of (MP).

Theorem 43 (First Epsilon Theorem for $\mathrm{EC}_{\varepsilon}$ ). If $E$ is a formula not containing any $\varepsilon$-terms and $\vdash_{\varepsilon} E$, then $\vdash_{\varepsilon} E$.

Proof. By induction on $o(\pi, r)$, we have: if $\vdash_{\varepsilon}^{\pi} E$, then there is a proof $\pi^{*}$ of $E$ with $\operatorname{rk}\left(\pi^{-}\right)<r$. By induction on $\operatorname{rk}(() \pi)$ we have a proof $\pi^{* *}$ of $E$ with $\operatorname{rk}\left(\pi^{* *}\right)=0$, i.e., without critical formulas at all.

Corollary 44 (Extended First $\varepsilon$-Theorem). If $\vdash_{\varepsilon} E\left(e_{1}, \ldots, e_{n}\right)$, then $\vdash_{\bigvee_{i=1}^{m}}^{m}$ $E\left(t_{1}^{j}, \ldots, t_{n}^{j}\right)$ for some terms $t_{j}$ (in $\left.\mathrm{EC}\right)$.

Proof. If $E$ contains $\varepsilon$-terms, say, $E$ is $E\left(e_{1}, \ldots, e_{n}\right)$, then replacement of $\varepsilon$ terms in the construction of $\pi_{i}$ may change $E$-but of course only the $\varepsilon$-terms appearing as subterms in it. In each step we obtain not a proof of $E$ but of some disjunction of instances $E\left(e_{1}^{\prime}, \ldots, e_{n}^{\prime}\right)$. For details, see [18].

\subsection{The Case with Identity}

In the presence of the identity $(=)$ predicate in the language, things get a bit more complicated. The reason is that instances of the $\left(=_{2}\right)$ axiom schema,

$$
t=u \rightarrow(A(t) \rightarrow A(u))
$$

may also contain $\varepsilon$-terms, and the replacement of an $\varepsilon$-term $e$ by a term $t_{i}$ in the construction of $\pi_{i}$ may result in a formula which no longer is an instance of $(=2)$. For instance, suppose that $t$ is a subterm of $e=e^{\prime}(t)$ and $A(t)$ is of the form $A^{\prime}\left(e^{\prime}(t)\right)$. Then the original axiom is

$$
t=u \rightarrow\left(A^{\prime}\left(e^{\prime}(t)\right) \rightarrow A^{\prime}\left(e^{\prime}(u)\right)\right.
$$

which after replacing $e=e^{\prime}(t)$ by $t_{i}$ turns into

$$
t=u \rightarrow\left(A^{\prime}\left(t_{i}\right) \rightarrow A^{\prime}\left(e^{\prime}(u)\right) .\right.
$$

So this must be avoided. In order to do this, we first observe that just as in the case of the predicate calculus, the instances of $\left(=_{2}\right)$ can be derived from restricted instances. In the case of the predicate calculus, the restricted axioms are

$$
\begin{aligned}
& t=u \rightarrow\left(P^{n}\left(s_{1}, \ldots, t, \ldots s_{n}\right) \rightarrow P^{n}\left(s_{1}, \ldots, u, \ldots, s_{n}\right)\right. \\
& t=u \rightarrow f^{n}\left(s_{1}, \ldots, t, \ldots, s_{n}\right)=f^{n}\left(s_{1}, \ldots, u, \ldots, s_{n}\right)
\end{aligned}
$$


to which we have to add the $\varepsilon$-identity axiom schema:

$$
t=u \rightarrow \varepsilon_{x} A\left(x ; s_{1}, \ldots, t, \ldots s_{n}\right)=\varepsilon_{x} A\left(x ; s_{1}, \ldots, u, \ldots s_{n}\right) \quad\left(={ }_{\varepsilon}\right)
$$

where $\varepsilon_{x} A\left(x ; x_{1}, \ldots, x_{n}\right)$ is an $\varepsilon$-type.

Proposition 45. Every instance of $\left(=_{2}\right)$ can be derived from $\left(=_{2}^{\prime}\right),\left(=_{2}^{\prime \prime}\right)$, and $(=\varepsilon)$.

Proof. By induction.

Now replacing every occurrence of $e$ in an instance of $\left(=_{2}^{\prime}\right)$ or $\left(=_{2}^{\prime \prime}\right)$-where $e$ obviously can only occur inside one of the terms $t, u, s_{1}, \ldots, s_{n}$-results in a (different) instance of $\left(=_{2}^{\prime}\right)$ or $\left(=_{2}^{\prime \prime}\right)$. The same is true of $\left(=_{\varepsilon}\right)$, provided that the $e$ is neither $\varepsilon_{x} A\left(x ; s_{1}, \ldots, t, \ldots s_{n}\right)$ nor $\varepsilon_{x} A\left(x ; s_{1}, \ldots, u, \ldots s_{n}\right)$. This would be guaranteed if the type of $e$ is not $\varepsilon_{x} A\left(x ; x_{1}, \ldots, x_{n}\right)$, in particular, if the rank of $e$ is higher than the rank of $\varepsilon_{x} A\left(x ; x_{1}, \ldots, x_{n}\right)$. Moreover, the result of replacing $e$ by $t_{i}$ in any such instance of $\left(=_{\varepsilon}\right)$ results in an instance of $\left(=_{\varepsilon}\right)$ which belongs to the same $\varepsilon$-type. Thus, in order for the proof of the first $\varepsilon$-theorem to work also when $=$ and axioms $\left(=_{1}\right),\left(=_{2}^{\prime}\right),\left(=_{2}^{\prime \prime}\right)$, and $\left(=_{\varepsilon}\right)$ are present, it suffices to show that the instances of $\left(=_{\varepsilon}\right)$ with $\varepsilon$-terms of $\operatorname{rank} \operatorname{rk}(\pi)$ can be removed. Call an $\varepsilon$-term $e$ special in $\pi$, if $\pi$ contains an occurrence of $t=u \rightarrow e^{\prime}=e$ as an instance of $\left(==_{\varepsilon}\right)$.

Theorem 46. If $\vdash_{\varepsilon=}^{\pi} E$, then there is a proof $\pi^{=}$so that $\vdash_{\varepsilon=}^{\pi^{=}} E, \operatorname{rk}\left(\pi^{=}\right)=$ $\operatorname{rk}(p i)$, and the rank of the special $\varepsilon$-terms in $\pi^{=}$has $\operatorname{rank}<\operatorname{rk}(\pi)$.

Proof. The basic idea is simple: Suppose $t=u \rightarrow e^{\prime}=e$ is an instance of $\left(={ }_{\varepsilon}\right)$, with $e^{\prime} \equiv \varepsilon_{x} A\left(x ; s_{1}, \ldots, t, \ldots s_{n}\right)$ and $e \equiv \varepsilon_{x} A\left(x ; s_{1}, \ldots, u, \ldots s_{n}\right)$. Replace $e$ everywhere in the proof by $e^{\prime}$. Then the instance of $\left(=_{\varepsilon}\right)$ under consideration is removed, since it is now provable from $e^{\prime}=e^{\prime}$. This potentially interferes with critical formulas belonging to $e$, but this can also be fixed: we just have to show that by a judicious choice of $e$ it can be done in such a way that the other $(=\varepsilon)$ axioms are still of the required form.

Let $p=\varepsilon_{x} A\left(x ; x_{1}, \ldots, x_{n}\right)$ be an $\varepsilon$-type of $\operatorname{rank} \operatorname{rk}(\pi)$, and let $e_{1}, \ldots, e_{l}$ be all the $\varepsilon$-terms of type $p$ which have a corresponding instance of $\left(=_{\varepsilon}\right)$ in $\pi$. Let $T_{i}$ be the set of all immediate subterms of $e_{1}, \ldots, e_{l}$, in the same position as $x_{i}$, i.e., the smallest set of terms so that if $e_{i} \equiv \varepsilon_{x} A\left(x ; t_{1}, \ldots, t_{n}\right)$, then $t_{i} \in T$. Now let let $T^{*}$ be all instances of $p$ with terms from $T_{i}$ substituted for the $x_{i}$. Obviously, $T$ and thus $T^{*}$ are finite (up to renaming of bound variables). Pick a strict order $\prec$ on $T$ which respects degree, i.e., if $\operatorname{deg}(t)<\operatorname{deg}(u)$ then $t \prec u$. Extend $\prec$ to $T^{*}$ by

$$
\varepsilon_{x} A\left(x ; t_{1}, \ldots, t_{n}\right) \prec \varepsilon_{x} A\left(x ; t_{1}^{\prime}, \ldots, t_{n}^{\prime}\right)
$$

iff

1. $\max \left\{\operatorname{deg}\left(t_{i}\right): i=1, \ldots, n\right\}<\max \left\{\operatorname{deg}\left(t_{i}\right): i=1, \ldots, n\right\}$ or 
2. $\max \left\{\operatorname{deg}\left(t_{i}\right): i=1, \ldots, n\right\}=\max \left\{\operatorname{deg}\left(t_{i}\right): i=1, \ldots, n\right\}$ and

(a) $t_{i} \equiv t_{i}^{\prime}$ for $i=1, \ldots, k$.

(b) $t_{k+1} \prec t_{k+1}^{\prime}$

Lemma 47. Suppose $\vdash_{\varepsilon=}^{\pi}$ E, e a special $\varepsilon$-term in $\pi$ with $\operatorname{rk}(e)=\operatorname{rk}(\pi), \operatorname{deg}(e)$ maximal among the special $\varepsilon$-terms of rank $\operatorname{rk}(\pi)$, and e maximal with respect to $\prec$ defined above. Let $t=u \rightarrow e^{\prime}=e$ be an instance of $\left(=_{\varepsilon}\right)$ in $\pi$. Then there is a proof $\pi^{\prime}, \vdash_{\varepsilon=}^{\pi^{\prime}} E$ such that

1. $\operatorname{rk}\left(\pi^{\prime}\right)=\operatorname{rk}(\pi)$

2. $\pi^{\prime}$ does not contain $t=u \rightarrow e^{\prime}=e$ as an axiom

3. Every special $\varepsilon$-term $e^{\prime \prime}$ of $\pi^{\prime}$ with the same type as $e$ is so that $e^{\prime \prime} \prec e$.

Proof. Let $\pi_{0}=\pi\left\{e / e^{\prime}\right\}$ and suppose $t^{\prime}=u^{\prime} \rightarrow e^{\prime \prime \prime}=e^{\prime \prime}$ is an $\left(=_{\varepsilon}\right)$ axiom in $\pi$.

If $\operatorname{rk}\left(e^{\prime \prime}\right)<\operatorname{rk}(e)$, then the replacement of $e$ by $e^{\prime}$ can only change subterms of $e^{\prime \prime}$ and $e^{\prime \prime \prime}$. In this case, the uniform replacement results in another instance of $\left(=_{\varepsilon}\right)$ with $\varepsilon$-terms of the same $\varepsilon$-type, and hence of the same $\operatorname{rank}<\operatorname{rk}(\pi)$, as the original.

If $\operatorname{rk}\left(e^{\prime \prime}\right)=\operatorname{rk}(e)$ but has a different type than $e$, then this axiom is unchanged in $\pi_{0}$ : Neither $e^{\prime \prime}$ nor $e^{\prime \prime \prime}$ can be $\equiv e$, because they have different $\varepsilon$-types, and neither $e^{\prime \prime}$ nor $e^{\prime \prime \prime}$ (nor $t^{\prime}$ or $u^{\prime}$, which are subterms of $e^{\prime \prime}, e^{\prime \prime \prime}$ ) can contain $e$ as a subterm, since then $e$ wouldn't be degree-maximal among the special $\varepsilon$-terms of $\pi$ of $\operatorname{rank} \operatorname{rk}(\pi)$.

If the type of $e^{\prime \prime}, e^{\prime \prime \prime}$ is the same as that of $e, e$ cannot be a proper subterm of $e^{\prime \prime}$ or $e^{\prime \prime \prime}$, since otherwise $e^{\prime \prime}$ or $e^{\prime \prime \prime}$ would again be a special $\varepsilon$-term of $\operatorname{rank} \operatorname{rk}(\pi)$ but of higher degree than $e$. So either $e \equiv e^{\prime \prime}$ or $e \equiv e^{\prime \prime \prime}$, without loss of generality suppose $e \equiv e^{\prime \prime}$. Then the $\left(=_{\varepsilon}\right)$ axiom in question has the form

$$
t^{\prime}=u^{\prime} \rightarrow \underbrace{\varepsilon_{x} A\left(x ; s_{1}, \ldots t^{\prime}, \ldots s_{n}\right)}_{e^{\prime \prime \prime}}=\underbrace{\varepsilon_{x} A\left(x ; s_{1}, \ldots u^{\prime}, \ldots s_{n}\right)}_{e^{\prime \prime} \equiv e}
$$

and with $e$ replaced by $e^{\prime}$ :

$$
t^{\prime}=u^{\prime} \rightarrow \underbrace{\varepsilon_{x} A\left(x ; s_{1}, \ldots t^{\prime}, \ldots s_{n}\right)}_{e^{\prime \prime \prime}}=\underbrace{\varepsilon_{x} A\left(x ; s_{1}, \ldots t, \ldots s_{n}\right)}_{e^{\prime}}
$$

which is no longer an instance of $\left(=_{\varepsilon}\right)$, but can be proved from new instances of $(=\varepsilon)$. We have to distinguish two cases according to whether the indicated position of $t$ and $t^{\prime}$ in $e^{\prime}, e^{\prime \prime \prime}$ is the same or not. In the first case, $u \equiv u^{\prime}$, and the new formula

$$
t^{\prime}=u \rightarrow \underbrace{\varepsilon_{x} A\left(x ; s_{1}, \ldots t^{\prime}, \ldots s_{n}\right)}_{e^{\prime \prime \prime}}=\underbrace{\varepsilon_{x} A\left(x ; s_{1}, \ldots t, \ldots s_{n}\right)}_{e^{\prime}}
$$

can be proved from $t=u$ together with

$$
\begin{aligned}
& t^{\prime}=t \rightarrow \underbrace{\varepsilon_{x} A\left(x ; s_{1}, \ldots t^{\prime}, \ldots s_{n}\right)}_{e^{\prime \prime \prime}}=\underbrace{\varepsilon_{x} A\left(x ; s_{1}, \ldots t, \ldots s_{n}\right)}_{e^{\prime}} \quad\left(={ }_{\varepsilon}\right) \\
& t=u \rightarrow\left(t^{\prime}=u \rightarrow t^{\prime}=t\right)
\end{aligned}
$$


Since $e^{\prime}$ and $e^{\prime \prime \prime}$ already occurred in $\pi$, by assumption $e^{\prime}, e^{\prime \prime \prime} \prec e$.

In the second case, the original formulas read, with terms indicated:

$$
\begin{aligned}
t & =u \rightarrow \underbrace{\varepsilon_{x} A\left(x ; s_{1}, \ldots t, \ldots, u^{\prime}, \ldots, s_{n}\right)}_{e^{\prime}}=\underbrace{\varepsilon_{x} A\left(x ; s_{1}, \ldots u, \ldots, u^{\prime}, \ldots, s_{n}\right)}_{e} \\
t^{\prime} & =u^{\prime} \rightarrow \underbrace{\varepsilon_{x} A\left(x ; s_{1}, \ldots u, \ldots, t^{\prime}, \ldots, s_{n}\right)}_{e^{\prime \prime \prime}}=\underbrace{\varepsilon_{x} A\left(x ; s_{1}, \ldots u, \ldots, u^{\prime}, \ldots, s_{n}\right)}_{e^{\prime \prime} \equiv e}
\end{aligned}
$$

and with $e$ replaced by $e^{\prime}$ the latter becomes:

$$
t^{\prime}=u^{\prime} \rightarrow \underbrace{\varepsilon_{x} A\left(x ; s_{1}, \ldots u, \ldots, t^{\prime}, \ldots s_{n}\right)}_{e^{\prime \prime \prime}}=\underbrace{\varepsilon_{x} A\left(x ; s_{1}, \ldots t, \ldots, u^{\prime}, \ldots, s_{n}\right)}_{e^{\prime}}
$$

This new formula is provable from $t=u$ together with

$$
\begin{gathered}
u=t \rightarrow \underbrace{\varepsilon_{x} A\left(x ; s_{1}, \ldots u, \ldots, t^{\prime}, \ldots s_{n}\right)}_{e^{\prime \prime \prime}}=\underbrace{\varepsilon_{x} A\left(x ; s_{1}, \ldots t, \ldots, t^{\prime}, \ldots, s_{n}\right)}_{e^{\prime \prime \prime \prime}} \\
t^{\prime}=u^{\prime} \rightarrow \underbrace{\varepsilon_{x} A\left(x ; s_{1}, \ldots t, \ldots, t^{\prime}, \ldots s_{n}\right)}_{e^{\prime \prime \prime \prime}}=\underbrace{\varepsilon_{x} A\left(x ; s_{1}, \ldots t, \ldots, u^{\prime}, \ldots, s_{n}\right)}
\end{gathered}
$$

and some instances of $\left(=_{2}^{\prime}\right)$. Hence, $\pi^{\prime}$ contains a (possibly new) special $\varepsilon$ term $e^{\prime \prime \prime \prime \prime}$. However, $e^{\prime \prime \prime \prime} \prec e$.

In the special case where $e=e^{\prime \prime}$ and $e^{\prime}=e^{\prime \prime \prime}$, i.e., the instance of $\left(=_{\varepsilon}\right)$ we started with, then replacing $e$ by $e^{\prime}$ results in $t=u \rightarrow e^{\prime}=e^{\prime}$, which is provable from $e^{\prime}=e^{\prime}$, an instance of $\left(=_{1}\right)$.

Let $\pi_{1}$ be $\pi_{0}$ with the necessary new instances of $\left(={ }_{\varepsilon}\right)$, added. The instances of $\left(=_{\varepsilon}\right)$ in $\pi_{1}$ satisfy the properties required in the statement of the lemma.

However, the results of replacing $e$ by $e^{\prime}$ may have impacted some of the critical formulas in the original proof. For a critical formula to which $e \equiv \varepsilon_{x} A(x, u)$ belongs is of the form

$$
A\left(t^{\prime}, u\right) \rightarrow A\left(\varepsilon_{x} A(x, u), u\right)
$$

which after replacing $e$ by $e^{\prime}$ becomes

$$
A\left(t^{\prime \prime}, u\right) \rightarrow A\left(\varepsilon_{x} A(x, t), u\right)
$$

which is no longer a critical formula. This formula, however, can be derived from $t=u$ together with

$$
\begin{aligned}
A\left(t^{\prime \prime}, u\right) & \rightarrow A\left(\varepsilon_{x} A(x, t), u\right) \\
t=u & \rightarrow\left(A\left(\varepsilon_{x} A(x, t), t\right) \rightarrow A\left(\varepsilon_{x} A(x, t), u\right)\right) \\
u=t & \rightarrow\left(A\left(t^{\prime \prime}, u\right) \rightarrow A\left(t^{\prime \prime}, t\right)\right)
\end{aligned}
$$

Let $\pi_{2}$ be $\pi_{1}$ plus these derivations of (2) with the instances of $\left(=_{2}\right)$ themselves proved from $\left(=_{2}^{\prime}\right)$ and $\left(=_{\varepsilon}\right)$. The rank of the new critical formulas is the same, 
so the rank of $\pi_{2}$ is the same as that of $\pi$. The new instances of $\left(=_{\varepsilon}\right)$ required for the derivation of the last two formulas only contain $\varepsilon$-terms of lower rank that that of $e$, as can be verified.

$\pi_{2}$ is thus a proof of $E$ from $t=u$ which satisfies the conditions of the lemma. From it, we obtain a proof $\pi_{2}[t=u]$ of $t=u \rightarrow E$ by the deduction theorem. On the other hand, the instance $t=u \rightarrow e^{\prime}=e$ under consideration can also be proved trivially from $t \neq u$. The proof $\pi[t \neq u]$ thus is also a proof, this time of $t \neq u \rightarrow E$, which satisfies the conditions of the lemma. We obtain $\pi^{\prime}$ by combining the two proofs.

Theorem 48 (First Epsilon Theorem for $\mathrm{EC}_{\varepsilon}^{=}$). If $E$ is a formula not containing any $\varepsilon$-terms and $\vdash_{\varepsilon=} E$, then $\vdash_{=} E\left(\right.$ in $\left.\mathrm{EC}^{=}\right)$.

Proof. By repeated application of the Lemma, every instance of $\left(=_{\varepsilon}\right)$ involving $\varepsilon$-terms of a given type $p$ can be eliminated from $\pi$. The Theorem follows by induction on the number of different types of special $\varepsilon$-terms of $\operatorname{rank} \operatorname{rk}(\pi)$ in $\pi$.

\section{Proof Theory of the Epsilon Calculus}

\subsection{Sequent Calculi}

Leisenring [12] presented a one-sided sequent calculus for the $\varepsilon$-calculus. It operates on sets of formulas (sequents); proofs are trees of sets of formulas each of which is either an axiom (at a leaf of the tree) or follows from the sets of formulas above it by an inference rule. Axioms are $A, \neg A$. The rules are given below:

$$
\begin{array}{lll}
\frac{\Gamma, A \quad \Gamma, B}{\Gamma, A \wedge B} \wedge R & \frac{\Gamma, \neg A, \neg B}{\Gamma, \neg(A \wedge B)} \wedge L & \left.\frac{\Gamma, A}{\Gamma, \neg \neg A} \neg\right\urcorner \\
\frac{\Gamma, A, B}{\Gamma, A \vee B} \vee R & \frac{\Gamma, \neg A \quad \Gamma, \neg B}{\Gamma, \neg(A \vee B)} \vee L & \frac{\Pi, A \quad \Lambda, \neg A}{\Pi, A} \text { cut } \\
\frac{\Gamma, A(t)}{\Gamma, \exists x A(x)} \exists R & \frac{\Gamma, \neg A\left(\varepsilon_{x} A(x)\right)}{\Gamma, \neg \exists x A(x)} \exists L & \frac{\Gamma, A}{\Gamma, A, B} w \\
\frac{\Gamma, A\left(\varepsilon_{x} \neg A(x)\right)}{\Gamma, \forall x A(x)} \forall R & \frac{\Gamma, \neg A(t)}{\Gamma, \neg \forall x A(x)} \forall L
\end{array}
$$

In contrast to classical sequent systems, there are no eigenvariable conditions!

It is complete, since proofs can easily be translated into derivations in $\mathrm{EC}_{\varepsilon}$; in particular it derives critical formulas:

$$
\frac{\frac{\neg A(t), A(t)}{\neg A(t), \sqrt{\exists x A(x)}} \exists R \frac{\neg A\left(\varepsilon_{x} A(x)\right), A\left(\varepsilon_{x} A(x)\right)}{\neg \exists x A(x), A\left(\varepsilon_{x} A(x)\right)}}{\neg L} \text { cut }
$$

This sequent, however, has no cut-free proof.

Maehara [13] instead proposed to simply add axioms corresponding to to critical formulas and leave out quantifier rules. Hence, its axioms are $\neg A, A$ and 
$\neg A(t), A\left(\varepsilon_{x} A(x)\right)$. It is complete, since the additional axioms allow derivation of critical formulas. However, it is also not cut-free complete. Converses of critical formulas are derivable using cut:

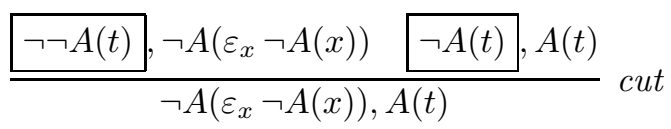

But these obviously have no cut-free proof. Furthermore, addition of these converses as axioms will not result in a cut-free complete system, either. Consider the example given by Wessels: Let $e=\varepsilon_{x} \neg(A(x) \vee B(x))$.

$$
\begin{aligned}
& \neg \neg(A(t) \vee B(t)), \neg(A(e) \vee B(e))
\end{aligned}
$$

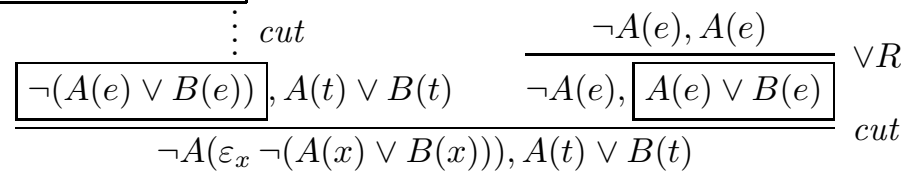

Wessels [20] proposed to add instead the following rule to the propositional one-sided sequent calculus:

$$
\frac{\Gamma, \Delta(z), \neg A(z) \quad \Gamma, A(t)}{\Gamma, \Delta\left(\varepsilon_{x} A(x)\right)} \varepsilon 0
$$

Here, $\Delta(z)$ must be not empty, and $z$ may not occur in the lower sequent. This system also derives critical formulas, and so is complete:

$$
\frac{\underbrace{\frac{A(z), \neg A(z)}{\neg A(t)}, \underbrace{A(a)}_{\Delta}, \neg A(z)}_{\Gamma} w \underbrace{\neg A(t)}_{\Gamma}, A(t)}{\neg A(t), A\left(\varepsilon_{x} A(x)\right)} \varepsilon 0
$$

The rule $\varepsilon 0$ is sound. ${ }^{3}$

Wessels offered a cut-elimination proof for her system. However, the proof relied on a false lemma to which Maehara gave a counterexample.

Wessels' Lemma. If $\vdash \Gamma, \Delta\left(\varepsilon_{x} A(x)\right)$ then $\vdash \Gamma, \Delta(z), \neg A(z)$.

Let $A(x)=P\left(x, \varepsilon_{y} Q\left(\varepsilon_{u} P(u, y)\right)\right), \Delta(z)=Q(z)$, and $\Gamma=\neg Q\left(\varepsilon_{x} B(x, w)\right)$. Then

$$
\underbrace{\neg Q\left(\varepsilon_{x} P(x, w)\right)}_{\Gamma}, \underbrace{Q\left(\varepsilon_{x} P\left(x, \varepsilon_{y} Q\left(\varepsilon_{u} P(u, y)\right)\right)\right)}_{\Delta\left(\varepsilon_{x} A(x)\right)}
$$

\footnotetext{
${ }^{3}$ Suppose the upper sequents are valid but the lower sequent is not, i.e., for some $\mathfrak{M}, \Psi, s, \mathfrak{M} \not \models \Gamma, \Delta\left(\varepsilon_{x} A(x)\right)$. In particular, $\mathfrak{M}, \Psi, s \not \models \Gamma$. Hence, $\mathfrak{M}, \Psi, s \models A(t)$, i.e., $\mathfrak{M}, \Psi, s \models A^{\prime}\left(t, t_{1}, \ldots, t_{n}\right)$, as the right premise is valid. So $\operatorname{val}_{\mathfrak{M}, \Psi, s}(t) \in$ $\operatorname{val}_{\mathfrak{M}, \Psi, s}(A(x))$. Now let $s(z)=\operatorname{val}_{M, \Psi, s}\left(\varepsilon_{x} A^{\prime}\left(x, t_{1}, \ldots, t_{n}\right)\right)$. Then $\mathfrak{M}, \Psi, s \models A(z)$ and so $\mathfrak{M}, \Psi, s \not \models \neg A(z)$. Since the left premise is valid, $\mathfrak{M}, \Psi, s \models \Delta(z)$. But also $\mathfrak{M}, \Psi, s \not \forall \Delta(z)$ since $\mathfrak{M}, \Psi, s \not \models \Delta\left(\varepsilon_{x} A(x)\right)$.
} 
is derivable, since it is of the form $\neg B(w), B\left(\varepsilon_{y} B(y)\right)$. However, the corresponding sequent in the consequent of the lemma,

$$
\underbrace{\neg Q\left(\varepsilon_{x} P(x, w)\right)}_{\Gamma}, \underbrace{Q(z)}_{\Delta(z)}, \underbrace{\neg P\left(z, \varepsilon_{y} Q\left(\varepsilon_{u} P(u, y)\right)\right)}_{\neg A(z)}
$$

is not derivable, because not valid. ${ }^{4}$

Mints (in a review of Wessels' paper) proposed the following rule instead:

$$
\frac{\Gamma, \Delta\left(\varepsilon_{x} A(x)\right), \neg A\left(\varepsilon_{x} A(x)\right) \quad \Gamma, A(t)}{\Gamma, \Delta\left(\varepsilon_{x} A(x)\right)} \varepsilon 1
$$

It, too, derives all critical formulas:

$$
\frac{\underbrace{\frac{A\left(\varepsilon_{x} A(x)\right), \neg A\left(\varepsilon_{x} A(x)\right)}{\neg A(t)}, \underbrace{A\left(\varepsilon_{x} A(x)\right)}_{\Delta}, \neg A\left(\varepsilon_{x} A(x)\right)}_{\Gamma} w \underbrace{\neg A(t), A\left(\varepsilon_{x} A(x)\right)}_{\Gamma}, A(t)}{\neg 1}
$$

The system was developed in detail by Yasuhara [21]. The Mints-Yasuhara system is cut-free complete. However, it is not known if the sequent has a cutelimination theorem that transforms a proof with cuts successively into one without cuts. Both Gentzen's and Tait's approach to cut-elimination do not seem to work. In a Gentzen-style proof, the main induction is on on cut length, i.e., the height of the proof tree above an uppermost cut. In the induction step, a cut is permuted upward to reduce the cut length. For instance, we replace the subproof proof ending in a cut

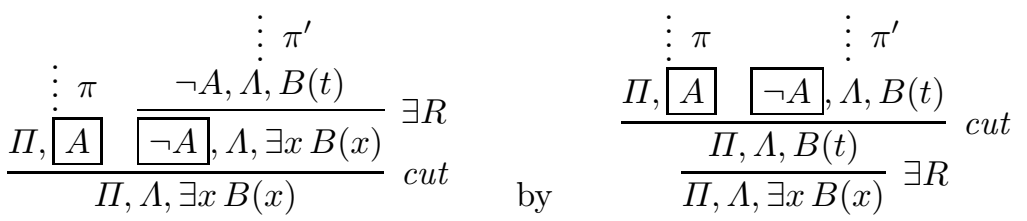

To permute a cut across the $\varepsilon 1$ rule:

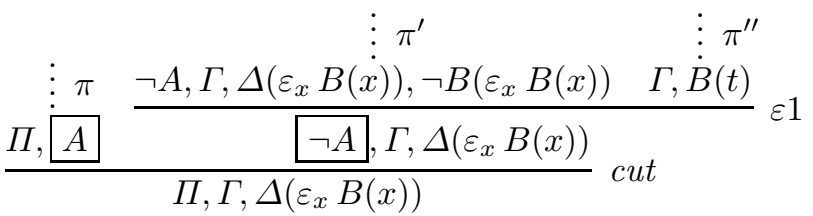

\footnotetext{
$\overline{{ }^{4} \text { Let }|\mathfrak{M}|=}\{1,2\}, Q^{\mathfrak{M}}=\{1\}, P^{\mathfrak{M}}=\{\langle 1,2\rangle,\langle 2,2\rangle\}, s(z)=s(w)=2$. Since $\langle 1,2\rangle \in$ $P^{\mathfrak{M}}$, we can choose $\Psi$ so that $\operatorname{val}_{\mathfrak{M}, \Psi, s}\left(\varepsilon_{x} P(x, 2)\right)=1$. So $\mathfrak{M}, \Psi, s \not \models \neg Q\left(\varepsilon_{x} P(x, w)\right)$. Also, $\mathfrak{M}, \Psi, s \not \models Q(z)$. As $\operatorname{val}_{\mathfrak{M}, \Psi, s}\left(\varepsilon_{u} P(u, 2)\right)=1$ and $1 \in Q^{\mathfrak{M}}$, we can also fix $\Psi$ so that $\operatorname{val}_{\mathfrak{M}, \Psi, s}\left(\varepsilon_{y} Q\left(\varepsilon_{u} P(u, y)\right)\right)=2$. But then $\mathfrak{M}, \Psi, s \not \models \neg P\left(z, \varepsilon_{y} Q\left(\varepsilon_{u} P(u, y)\right)\right)$.)
} 
one might try to replace the proof tree with

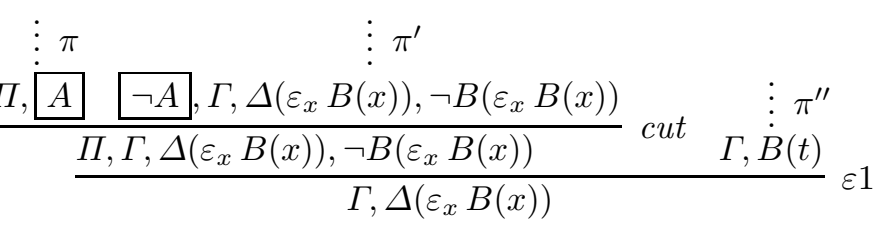

However, here the condition on $\varepsilon 1$ is violated if $\neg A$ is in $\Delta$.

In a Tait-style cut elimination proof, the main induction is on cut rank, i.e., complexity of the cut formula. In the induction step, the complexity of the cut formula is reduced. For instance, if a subproof ends in a cut

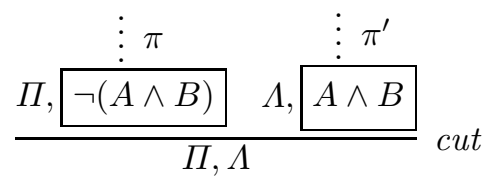

we replace it with

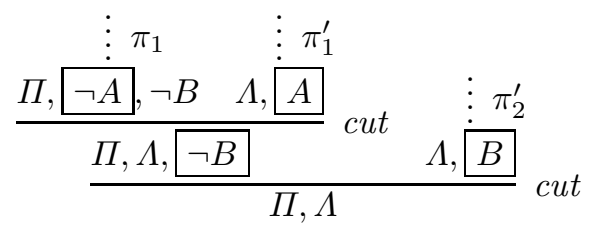

This approach requires inversion lemmas. A typical case is: If $\pi^{\prime} \vdash \Pi, A \wedge B$ then there is a $\pi_{1}^{\prime} \vdash \Pi, A$ of cut rank and length $\leq$ that of $\pi^{\prime}$. In the proof of the inversion lemma, one replaces all ancestors of $A \wedge B$ in $\pi^{\prime}$ by $A$ and "fixes" those rules that are no longer valid. For instance, replace

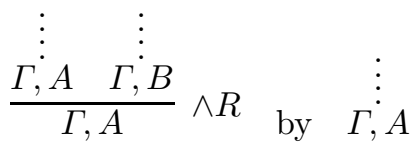

But now consider a derivation $\pi^{\prime}$ which contains the $\varepsilon 1$ rule: ${ }^{5}$

$$
\begin{array}{cc}
\vdots & \vdots \\
\Pi, A \wedge B\left(\varepsilon_{x} C(x)\right), \neg C\left(\varepsilon_{x} C(x)\right) & \Pi, \dot{C}(t) \\
\Pi, A \wedge B\left(\varepsilon_{x} C(x)\right. &
\end{array}
$$

The inversion lemma produces

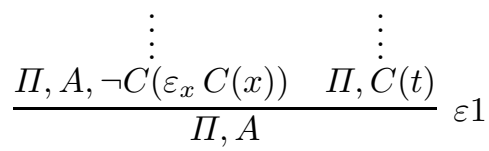

This, again, no longer satisfies the condition of $\varepsilon_{1}$.

${ }^{5}\left(A \wedge B\left(\varepsilon_{x} C(x)\right)\right.$ is $\Delta\left(\varepsilon_{x} C(x)\right)$ in this case). 
Open Problem 49. Prove cut-elimination for the Mints-Yasuhara system, or give a similarly simple sequent calculus for which it can be proved.

\subsection{Natural deduction}

In Gentzen's classical natural deduction system NK, the quantifier rules are given by

$$
\begin{array}{cc}
\frac{A(z)}{\forall x A(x)} \forall I & \frac{\forall x A(x)}{A(t)} \forall E \\
& {[A(z)]} \\
\vdots & \\
\frac{A(t)}{\exists x A(x)} \exists I & \frac{\exists x A(x) \quad \dot{C}}{B} \exists E
\end{array}
$$

where $z$ must not appear in any undischarged assumptions (nor in $A(x)$ or $B$ ). Meyer Viol [15] has proposed a system in which the $\exists E$ rule is replaced by

$$
\frac{\exists x A(x)}{A\left(\varepsilon_{x} A(x)\right)} \exists E_{\varepsilon}
$$

and the following term rule is added

$$
\frac{A(t)}{A\left(\varepsilon_{x} A(x)\right)} I \varepsilon
$$

Open Problem 50. Does Meyer Viol's system have a normal form theorem?

Adding $\exists E_{\varepsilon}$ and $I \varepsilon$ to the intuitionistic system NJ results in a system that is not conservative over intuitionistic logic. For instance, Plato's principle, the formula

$$
\exists x(\exists y A(y) \rightarrow A(x))
$$

becomes derivable:

$$
\frac{\frac{\left[\exists y A\left(\varepsilon_{x} A(x)\right)\right]}{A\left(\varepsilon_{x} A(x)\right)} \exists E_{\varepsilon}}{\frac{\exists y A(y) \rightarrow A\left(\varepsilon_{x} A(x)\right)}{\exists x(\exists y A(y) \rightarrow A(x))} \rightarrow I} \exists I
$$

However, the system also does not collapse to classical logic: it is conservative for propositional formulas.

Intuitionistic natural deduction systems are especially intriguing, as Abadi, Gonthier and Werner [1] have shown that a system of quantified propositional intuitionistic logic with a choice operator $\varepsilon_{X}$ can be given a Curry-Howard correspondence via a type system which $\varepsilon_{X} A(X)$ is a type such that the type $A(X)$ is inhabited. System $\mathcal{E}$ is paired with a simply typed $\lambda$-calculus that, in addition to $\lambda$-abstraction and application, features implementation: $\langle t: A$ with $X=T\rangle$ of type $A\left(\epsilon_{X} A / X\right)$. If $A(X)$ is a type specification of an interface with variable type $X$, then $A(T)$ for some type $T$ is an implementation of that interface. 


\section{References}

1. Abadi, M., Gonthier, G., Werner, B.: Choice in dynamic linking. In: Foundations of Software Science and Computation Structures FOSSACS 2004. pp. 12-26. No. 2987 in LNCS, Springer, Berlin (2004)

2. Abiteboul, S., Vianu, V.: Non-determinism in logic-based languages. Annals of Mathematics and Artificial Intelligence 3(2-4), 151-186 (1991)

3. Asser, G.: Theorie der logischen Auswahlfunktionen. Z. Math. Logik Grundlag. Math. 3, 30-68 (1957)

4. Avigad, J., Zach, R.: The Epsilon Calculus. In: Zalta, E.N. (ed.) The Stanford Encyclopedia of Philosophy (Summer 2016 Edition) (2016), http://plato.stanford.edu/entries/epsilon-calculus/

5. Bell, J.L.: Hilbert's epsilon-operator and classical logic. J. Philos. Logic 22, 1-18 (1993)

6. Blass, A., Gurevich, Y.: The logic of choice. Journal of Symbolic Logic 65, 1264$1310(2000)$

7. DeVidi, D.: Intuitionistic epsilon- and tau-calculi. Mathematical Logic Quarterly 41, 523-546 (1995)

8. von Heusinger, K.: The reference of indefinites. In: von Heusinger, K., Egli, U. (eds.) Reference and Anaphoric Relations, pp. 247-25. Kluwer, Dordrecht (2000)

9. von Heusinger, K.: Choice functions and the anaphoric semantics of definite nps. Research in Language and Computation 2, 309-329 (2004)

10. Hilbert, D.: Neubegründung der Mathematik: Erste Mitteilung. Abhandlungen aus dem Seminar der Hamburgischen Universität 1, 157-77 (1922), series of talks given at the University of Hamburg, July 25-27, 1921. English in [14], pp. 198-214

11. Hilbert, D., Bernays, P.: Grundlagen der Mathematik, vol. 2. Springer, Berlin (1939)

12. Leisenring, A.: Mathematical Logic and Hilbert's $\epsilon$-symbol. MacDonald Technical and Scientific, London (1969)

13. Maehara, S.: The predicate calculus with $\epsilon$-symbol. Journal of the Mathematical Society of Japan 7, 323-344 (1955)

14. Mancosu, P. (ed.): From Brouwer to Hilbert. The Debate on the Foundations of Mathematics in the 1920s. Oxford University Press, New York and Oxford (1998)

15. Meyer Viol, W.P.M.: Instantial logic. An Investigation into Reasoning with Instances. ILLC Dissertation Series 1995-11, ILLC, Amsterdam (1995)

16. Mints, G., Sarenac, D.: Completeness of indexed epsilon-calculus. Archive for Mathematical Logic 42, 617-625 (2003)

17. Mints, G.: Heyting predicate calculus with epsilon symbol. Journal of Soviet Mathematics 8, 317-323 (1977)

18. Moser, G., Zach, R.: The epsilon calculus and herbrand complexity. Studia Logica 82(1), 133-155 (2006)

19. Otto, M.: Epsilon-logic is more expressive than first-order logic over finite structures. Journal of Symbolic Logic 65(4), 1749-1757 (2000)

20. Wessels, L.: Cut elimination in a Gentzen-style $\varepsilon$-calculus without identity. Z. Math. Logik Grundlag. Math. 23, 527-538 (1977)

21. Yashahura, M.: Cut elimination in $\epsilon$-calculi. Z. Math. Logik Grundlag. Math. 28, 311-316 (1982) 